\title{
Importance of a Blood Test Before Starting a Protein Ketogenic Diet
}

\author{
Joaquin Perez-Guisado Rosa* \\ Department of Genetic, University of Córdoba, Campus of Rabanales, Córdoba
}

\begin{abstract}
Received: : June 28, 2015; Accepted: July 20, 2015; Published: September 20, 2015
*Corresponding author: Joaquín Pérez-Guisado Rosa, MD, Ph.D, Department of Genetic, University of Córdoba, Campus of Rabanales, Córdoba, Spain. E-mail: pv1peguj@uco.es
\end{abstract}

As we all know, obesity is not only an aesthetic problem, but in developed countries, it is a disease that is reaching epidemic proportions and is associated with a multitude of medical conditions. Few examples of scientifically proven and its association with, are given below:

\section{A lower life expectancy}

2. A higher incidence with worse prognosis and evolution in different types of cancer such as the esophagus, stomach, colon, breast, endometrium, ovary, kidney and pancreas.

3. Cardiovascular problems or diseases associated such as dyslipidemia, hypertension, peripheral vascular disease, phlebitis and venous thromboembolic disease.

4. Endocrine problems where resistance to insulin, either directly or indirectly plays a key role, as the metabolic syndrome, impaired fasting glucose, carbohydrate intolerance, type 2 diabetes mellitus and polycystic ovary syndrome.

5. Both male and female infertility and menstrual disorders

6. Orthopedic problems such as osteoarthritis.

7. Hyperuricemia and gout.

8. Chronic kidney disease due to glomerular damage.

9. Gastroesophageal reflux disease and hiatal hernia.

10. Vision problems such as glaucoma, macular degeneration and cataracts

11. Liver problems such as gallstones and liver steatosis, closely related to insulin resistance.

12. Respiratory problems such as dyspnea, sleep apnea and asthma.

13. Psychological problems such as low self-esteem, depression and anxiety.

A protein ketogenic diet is a ketogenic diet based on proteins. I have treated successfully with this diet, mainly with the "Spanish ketogenic Mediterranean diet" to more than 100 obese patients.
Moreover, my Ph.D is based on this diet. For this reason, I would like to give advice based on my experience concerning this diet and specifically related to the importance of the blood test before starting this diet.

Considered obesity as a disease per se and its possible association with other comorbidities, the medical consultation is very important. This checkup should include a complete medical history to consider personal and family history and physical examination that takes into account the patient's weight, height, waist and hip circumference measurement and blood pressure. The blood test has a great importance, because on one hand it is not only important to know the external physical condition, but also we will know how our patient is regarding internal health markers, and how they evolve conducting diet and subsequent weight loss. Through the blood test, we can also determine whether the patient has any medical problem that could dissuade us from performing the ketogenic diet or could recommend us the use of any medication or additional measures. In those cases where we think that obesity could have an endocrine background, a more exhaustive blood test, also allow us to rule out or detect possible endocrine pathologies associated with it.

Given all this, a complete blood test before conducting a protein ketogenic diet should include:

1. The hemogram and electrolyte profile that consider the sodium, potassium, magnesium, calcium and chlorine. We all know that obesity is not synonymous of eating well or balanced, so could detect deficiencies as may be iron deficiency (microcytic anemia) of folic acid and vitamin B12 even (macrocytic anemias) in our obese patients. We should have in mind these alterations in order to focus on them appropriately so we don't worse them with the completion of the ketogenic diet. Concerning the ions indicated, they are important to know if the patient has some sort of predisposition to electrolyte abnormalities that become apparent in the blood sample and that could exacerbate when the patient start the ketogenic diet, so we could act and prevent through the proper supplementation.

2. A lipid profile by determining triglyceride, total cholesterol, 
LDL and HDL. In obese patients, we find very often an altered lipid profile, presenting elevated triglycerides, total cholesterol and $L D L$ and low levels of HDL. With the ketogenic diet, we usually find in the next monthly blood test an improvement in all these parameters, this is due not only to the weight loss, but also to an improvement in insulin sensitivity. As in everything, there are exceptional cases where there could be a worsening of the lipid profile, as could be the Euthyroid Sick Syndrome (ESS), Sick Euthyroid Syndrome (SES) or Non-Thyroidal Illness Syndrome (NTIS), which is currently considered as the most common cause of alteration in plasma thyroid hormone and which could cause a typical dyslipidaemia of hypothyroidism. This alteration is a defense mechanism of our body in situations where it is necessary to consider energy savings, appearing in any patients with nonthyroidal acute or chronic diseases, in postoperative states, fasting, malnutrition or important caloric restriction, which is very typical in the anorexic disorder. The body, to lower the basal, reduces the plasma concentration of T3 by 3 ways: decreasing the binding of T3 to the thyroid hormone transport protein (thereby decreasing the reserve of these hormones and increasing their metabolism speeds ), increasing the peripheral conversion of T4 to rT3 (inactive) and decreasing the conversion of T4 to T3 (metabolically the most active).

3. An analysis of glucose metabolism by determining the fasting glucose. In diabetic patients, if we want to know retrospectively how has been the control of their diabetes 2-3 months before the blood sample, we can determine glycosylated hemoglobin (HbA1c), which will present values below 7\% if the control has been right. In general, all patients that perform the ketogenic diet improve fasting blood glucose as a result of an improvement in insulin sensitivity.

4. An analysis of the liver and biliary function by assessing the bilirubin, ALT, AST, GGT and alkaline phosphatase. This is important because many obese patients may have problems especially cholelithiasis and fatty liver associated with the insulin resistance. Normally, the fatty liver doesn't have associated transaminase levels that go beyond twice the normal values and the ketogenic diet will normalize their values, although there may be an initial increase at the beginning of the diet. In those cases where we have particularly high levels of transaminases, we should do a further study because the liver problem is probably due to another entity. As for the question of what levels of maximum transaminase levels, we could include patients in the therapeutic protocol of the protein ketogenic diet, with up to three times their maximum values of transaminases, although the doctor will take the final decision and it is totally acceptable not to include a patient in the protocol if their values exceed twice than the normal values.

5. An analysis of renal function by measuring plasma urea and creatinine. These determinations are vital because renal failure is an absolute contraindication for the protein ketogenic diet.
Keep in mind that urea levels depend on several factors such as the type of food, the liver protein catabolism and diuresis. While creatinine levels are more related to muscle protein catabolism and diuresis. These considerations may be of importance to us in order to know if our patient has a renal disease, which will be one in which both plasma urea and creatinine values are presented above the normal levels and compliance that, the ratio of urea and creatinine is approximately $10 / 1$. We must be alert because it might be the case that due to a high protein diet, a high protein catabolism and even dehydration, determinations of urea and even creatinine could be above the normal levels without any renal failure associated, in this case we should find that, the urea and creatinine ratio exceeds 10 to 15 . Finally, we could be facing a patient in which the values of urea and creatinine or only creatinine are exceeding normal levels without there being either associated renal insufficiency, in this case, we could find the ratio of urea and creatinine less than 10 , it could be due to a liver disease, overhydration and even rhabdomyolysis.

6. Determination of uric acid, especially in patients with a history of gout or hyperuricemia. We should know that a protein ketogenic diet may increase in these patients the levels of this marker, being a relative contraindication when making this diet. If we have a patient with this problem, we could solve it through a previous normalization of uric acid by treatment with allopurinol for 2-3 weeks before starting the diet. Once the patient got such normalization, he could start the diet with the condition that he maintain the normal levels of uric acid with allopurinol treatment and we check it with regular blood sample tests (at least once a month).

7. In those cases where we think there might be an endocrine pathology associated we should discard two diseases: hypothyroidism and Cushing syndrome. In hypothyroidism, we determine the plasma concentration of TSH, T3 and T4, and we must never forget that the best biochemical marker for the diagnosis and management of hypothyroidism is the TSH, as it should always be high. In hypothyroidism, the most frequent is having high levels of TSH with low levels of T4 and T3, however when the hypothyroidism is subclinical, the TSH remains high while the T4 is normal or low and the T3 is normal. Concerning the Cushing syndrome, the two most widely used tests when there is a suspected diagnosis are: urinary free cortisol determination in 24-hour urine test or rapid dexamethasone suppression test. Rapid dexamethasone suppression test consist of giving the patient before bedtime $1 \mathrm{mg}$ of dexamethasone and determine the next morning plasma cortisol levels, which must be descended if the patient does not have Cushing Syndrome.

Finally, I would like to remember that we are responsible with our patients for their success in weight loss, and that we should make guidelines and follow a scientifically based protocol that produces in our patients a healthy weight loss. 